\title{
Dyslipidemia causes overestimation of plasma mitotane measurements
}

\author{
Angelo Paci1,*, Ségolène Hescot2,3,*, Atmane Seck', Christel Jublanc4, Lionel Mercier¹, Delphine Vezzosi5, \\ Delphine Drui 6 , Marcus Quinkler7, Martin Fassnacht8, Eric Bruckert4, Marc Lombès², Sophie Leboulleux ${ }^{3}$, \\ Sophie Broutin ${ }^{1}$ and Eric Baudin ${ }^{2,3}$
}

1Pharmacology and Drug Analysis Department, Gustave Roussy, Villejuif, France, 2INSERM U1185, Fac Med Paris Sud, Le Kremlin-Bicêtre, France, ${ }^{3}$ Nuclear Medicine and Endocrine Oncology Department, Gustave Roussy, Villejuif, France, ${ }^{4}$ Assistance Publique-Hôpitaux de Paris, La Pitié-Salpetriere Hospital, Department of Endocrinology, Paris, France, ${ }^{5} \mathrm{CHU}$ Larrey, Department of Endocrinology, Toulouse, France, ${ }^{6} \mathrm{CHU}$ Nantes, Department of Endocrinology, Nantes, France, ${ }^{7}$ Endocrinology in Charlottenburg, Berlin, Germany, and ${ }^{8}$ Endocrine and Diabetes Unit, Department of Medicine 1, University Hospital, University of Würzburg, Würzburg, Germany

*(A Paci and S Hescot contributed equally to this work)

Correspondence should be addressed to E Baudin

Email

eric.baudin@gustaveroussy.fr

\section{Summary}

Mitotane $\left(o, p^{\prime}\right.$-DDD) is the standard treatment for advanced adrenocortical carcinoma (ACC). Monitoring of plasma mitotane levels is recommended to look for a therapeutic window between 14 and $20 \mathrm{mg} / \mathrm{L}$, but its positive predictive value requires optimization. We report the case of an ACC patient with a history of dyslipidemia treated with mitotane in whom several plasma mitotane levels $>30 \mathrm{mg} / \mathrm{L}$ were found together with an excellent neurological tolerance. This observation led us to compare theoretical or measured $o, p^{\prime}$-DDD and $o, p^{\prime}$-DDE levels in a series of normolipidemic and dyslipidemic plasma samples to explore potential analytical issues responsible for an overestimation of plasma mitotane levels. We demonstrate an overestimation of mitotane measurements in dyslipidemic patients. Mitotane and $o, p^{\prime}$-DDE measurements showed a mean $20 \%$ overestimation in hypercholesterolemic and hypertriglyceridemic plasma, compared with normolipidemic plasma. The internal standard $p, p^{\prime}$-DDE measurements showed a parallel decrease in hypercholesterolemic and hypertriglyceridemic plasma, suggesting a matrix effect. Finally, diluting plasma samples and/or using phospholipid removal cartridges allowed correcting such interference.

\section{Learning points:}

- Hypercholesterolemia $(\mathrm{HCH})$ and hypertriglyceridemia (HTG) induce an overestimation of plasma mitotane measurements.

- We propose a routine monitoring of lipidemic status.

- We propose optimized methodology of measurement before interpreting high plasma mitotane levels.

\section{Background}

Monitoring plasma mitotane levels is recommended in the follow-up of patients with unresectable adrenocortical carcinoma (ACC) to look for a therapeutic window of $14-20 \mathrm{mg} / \mathrm{L}$ to optimize benefit over risk and avoid toxicities (1). Mitotane is a lipophilic drug that accumulates in lipoproteins and induces dyslipidemia (hypercholesterolemia $(\mathrm{HCH})$ and/or hypertriglyceridemia (HTG)) $(2,3,4,5)$. Previous studies suggested that 
the lipoprotein profile may influence mitotane drug distribution (6). Moreover, high plasma mitotane levels have been described in dyslipidemic patients who did not exhibit any side effects, suggesting either methodological issues, or that plasma mitotane distribution in lipoprotein subtypes is a major determinant of its distribution in tissues (7). Indeed, we recently reported that plasma mitotane level was correlated with $o, p^{\prime}$-DDD measured in HDL and LDL fractions (8).

Here, we report the case of an ACC patient with a severe dyslipidemia. Plasma mitotane monitoring revealed very high levels, that is, $>30 \mathrm{mg} / \mathrm{L}$, while our patient did not present any neurological side effects. Considering this observation, we demonstrate that dyslipidemia causes an overestimation of plasma mitotane levels explained by a so-called matrix effect.

\section{Case presentation}

Mr A, a 39-year-old man was referred in our institution in July 2007 for the therapeutic management of a functioning stage IV ACC with lung metastases. The main characteristics at the time of his first visit were a R0 ACC primary removal and a Weiss score of 6 including $<5$ mitosis per 50 high-power field (HPF). Previous history included obesity. During the 90-month of the follow-up period with mitotane therapy, 19 out of 103 plasma mitotane levels were found $>20 \mathrm{mg} / \mathrm{L}$ including $9>30 \mathrm{mg} / \mathrm{L}$ (up to $78 \mathrm{mg} / \mathrm{L}$ ). Several sequences of radiofrequency or cytotoxic chemotherapies were used. Best morphological response according to response evaluation criteria in solid tumours (RECIST) 1.0 criteria was stable disease at

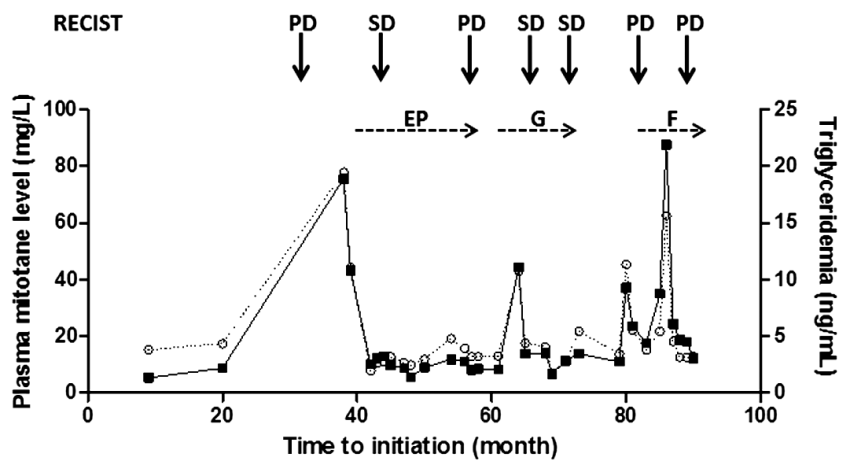

Figure 1

Monitoring of Mr A during mitotane therapy. Plasma mitotane level in $\mathrm{mg} / \mathrm{L}$ (white circles) and triglyceridemia in $\mathrm{ng} / \mathrm{mL}$ (black squares). Plasma mitotane measurements were performed using HPLC-UV; triglycerides measurements were performed using Unicel DXC Beckman Coulter Automate (Marseille, France). EP, etoposide-platin; F, fluorouracil; $\mathrm{G}$, gemcitabine; PD, progressive disease; SD, stable disease. the time of gemcitabine-based chemotherapy sequence as indicated in Fig. 1. Surprisingly, no single-grade 2-4 neurological adverse event was observed according to the National Cancer Institute Common Terminology Criteria for Adverse Events v4 (NCI-CTCAE) criteria. At 3 months of mitotane therapy, an increase in LDL-cholesterol was found and resolved after the introduction of statins in September 2008. From 2008 onward, several triglyceride measurements were performed at the time of evaluation of plasma mitotane level. As shown in Fig. 1, a narrow parallelism was observed between the two curves. This observation led us to explore potential analytical issues responsible for an overestimation of plasma mitotane levels in patients with dyslipidemia.

\section{Investigation}

\section{Influence of HCH and HTG on plasma $0, p^{\prime}-D D D$ and $o, p^{\prime}$-DDE measurements}

In routine practice, plasma mitotane concentrations are assayed by HPLC-UV using $p, p^{\prime}$-DDE as an internal standard (IS). Plasma samples from normolipidemic (cholesterol $<1 \mathrm{~g} / \mathrm{L}$ and triglyceride $<1 \mathrm{~g} / \mathrm{L}$ ) and dyslipidemic patients provided by various hospitals were spiked with fixed known concentrations of $o, p^{\prime}$-DDD, $o, p^{\prime}$-DDE, and $p, p^{\prime}$-DDE. Mitotane and its metabolite $o, p^{\prime}$-DDE concentrations were determined through the ratio of their peak surface area to the peak surface area of the known concentration of $p, p^{\prime}$-DDE. After a liquidliquid extraction, $o, p^{\prime}$-DDD and $o, p^{\prime}$-DDE measurements were determined and compared with the known theoretical concentrations. We showed that $\mathrm{HCH}$ (Fig. 2A) and HTG (Fig. 2B) led to an overestimation (from 10 to $75 \%$ ) of measured plasma mitotane levels as compared with theoretical concentrations. The overestimation was similar (from 5 to 66\%) for $o, p^{\prime}$-DDE, as shown in Fig. 2C and D. $o, p^{\prime}-\mathrm{DDE}$ is routinely performed at our institution for research purpose and was studied in an attempt to be exhaustive. By contrast, the measured concentration of $p, p^{\prime}$-DDE, used as internal standard, gradually decreased as the amount of $\mathrm{HCH}$ and HTG increased (Fig. 2E and F).

To confirm the matrix effect, $\mathrm{HCH}$ and HTG plasmas were first diluted with normolipidemic plasma without mitotane. Second, Phree phospholipid removal cartridges were used. In comparison with theoretical values, $p, p^{\prime}$-DDE values were found underestimated with a mean of $-21.1 \%$ in eight plasma samples with high levels of lipids. By contrast, same samples analyzed after Phree extraction were found similar to theoretical values (mean $=-2.3 \%)$. 

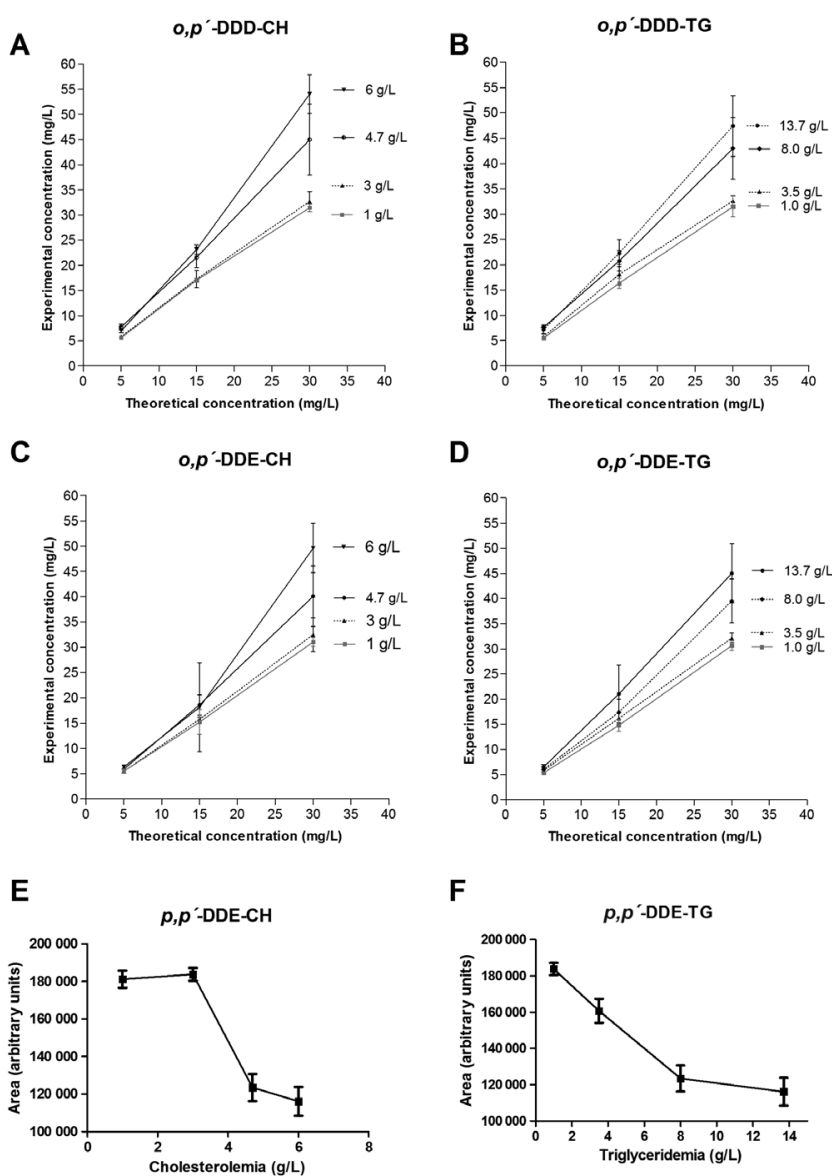

Figure 2

Impact of cholesterol concentration on measurements of plasma mitotane (o, $p^{\prime}$-DDD: $\left.2 A\right)$ and its metabolite $\left(o, p^{\prime}-\mathrm{DDE}: 2 \mathrm{C}\right)$ and the internal standard ( $\left.p, p^{\prime}-\mathrm{DDE}: 2 \mathrm{E}\right)$ in vitro. Impact of triglyceride concentration on measurements of plasma mitotane (o, $\left.p^{\prime}-\mathrm{DDD}: 2 \mathrm{~B}\right)$ and its metabolite (o, $p^{\prime}$-DDE: $\left.2 \mathrm{D}\right)$ and the internal standard ( $p, p^{\prime}$-DDE: $2 \mathrm{~F}$ ) in vitro. Measurements were performed in triplicate in each normal or dyslipidemic samples.

\section{Discussion}

We report for the first time an analytical overestimation of mitotane measurements in the plasma of dyslipidemic patients. From this case report, we hypothesized that a methodological issue could explain the absence of side effects in dyslipidemic patients with plasma mitotane levels $>30 \mathrm{mg} / \mathrm{L}$. Our study confirmed that mitotane and $o, p^{\prime}$-DDE measurements were systematically overestimated by $10 \%$ when $\mathrm{HCH}$ was at $3 \mathrm{~g} / \mathrm{L}$ and $>50 \%$ when $\mathrm{HCH}$ was at $6 \mathrm{~g} / \mathrm{L}$. This overestimation was similar in HTG plasma (3.5-13.7 g/L). In clinical practice, these results indicate that cholesterol and triglyceride concentrations should be analyzed together with mitotane measurements on a regular basis to improve the accuracy of plasma mitotane measurements for a given patient. Our results show that this so-called matrix effect phenomenon linked to a reduced extraction yield of $p, p^{\prime}$-DDE is responsible for this overestimation. Indeed, the decrease in the area under curve of $p, p^{\prime}$-DDE levels was proportional to the increases observed in $o, p^{\prime}$-DDD and $o, p^{\prime}$-DDE levels and normalized by diluting the plasma sample with normolipidemic plasma without mitotane or using phospholipid removal cartridges. At the end, mitotane-induced dyslipidemia may interfere with the tumor activity of the drug. Indeed, Hescot et al. previously supported that mitotane unbound to lipoprotein fractions is more efficient in vitro (8).

In conclusion, we demonstrate that $\mathrm{HCH}$ and/or HTG, a common side effect of mitotane, lead to an overestimation of its measurements suggesting that lipid alterations should be monitored in routine practice and optimized techniques should be implemented in patients with dyslipidemia.

\section{Declaration of interest}

The authors declare that there is no conflict of interest that could be perceived as prejudicing the impartiality of this research reported.

Funding

S H was recipient of a fellowship from HRA Pharma Laboratories (Bourse (IFRE).

Author contribution statement

A $P, S H$, and $E B$ designed the study. A $P, S H, A S, C J, L M$, and $S B$ performed experiments and analyzed the results. $A P, S H, D V, D D, M Q$, $M F, E B, M L, S L, S B$, and $E B$ contributed to interpretation of data and participated in discussion. $\mathrm{S} \mathrm{H}, \mathrm{A}$ P, and E B wrote the paper. All authors have read, revised, and approved the manuscript.

Acknowledgments

The authors are grateful to Lorna Saint-Ange for editing this manuscript.

\section{References}

1 Berruti A, Baudin E, Gelderblom H, Haak HR, Porpiglia F, Fassnacht M \& Pentheroudakis G 2012 Adrenal cancer: ESMO Clinical Practice Guidelines for diagnosis, treatment and follow-up. Annals of Oncology 23 (Supplement 7) vii131-vii138.

2 Gebhardt DO, Moolenaar AJ, van Seters AP, van der Velde EA \& Gevers Leuven JA 1992 The distribution of o,p'-DDD (mitotane) among serum lipoproteins in normo- and hypertriglyceridemia. Cancer Chemotherapy and Pharmacology 29 331-334. (doi:10.1007/BF00685956)

3 Daffara F, De Francia S, Reimondo G, Zaggia B, Aroasio E, Porpiglia F, Volante M, Termine A, Di Carlo F, Dogliotti L, et al. 2008 Prospective evaluation of mitotane toxicity in adrenocortical cancer patients treated adjuvantly. Endocrine-Related Cancer 15 1043-1053. (doi:10.1677/ERC-08-0103) 
4 Shawa H, Deniz F, Bazerbashi H, Hernandez M, Vassilopoulou-Sellin R, Jimenez C \& Habra MA 2013 Mitotane-induced hyperlipidemia: a retrospective cohort study. International Journal of Endocrinology 2013 624962.

5 Maher VM, Trainer PJ, Scoppola A, Anderson JV, Thompson GR \& Besser GM 1992 Possible mechanism and treatment of o,p'DDD-induced hypercholesterolaemia. Quarterly Journal of Medicine 84 671-679.

6 Pohland RC \& Counsell RE 1985 The role of high density lipoproteins in the biodistribution of two radioiodinated probes in the rat. Toxicology and Applied Pharmacology 77 47-57. (doi:10.1016/0041008X(85)90266-2)
7 Mauclère-Denost S, Leboulleux S, Borget I, Paci A, Young J, Al Ghuzlan A, Deandreis D, Drouard L, Tabarin A, Chanson P, et al. 2012 High-dose mitotane strategy in adrenocortical carcinoma: prospective analysis of plasma mitotane measurement during the first 3 months of follow-up. European Journal of Endocrinology 166 261-268. (doi:10.1530/EJE-11-0557)

8 Hescot S, Seck A, Guerin M, Cockenpot F, Huby T, Broutin S, Young J, Paci A, Baudin E \& Lombès M 2015 Lipoprotein-free mitotane exerts high cytotoxic activity in adrenocortical carcinoma. Journal of Clinical Endocrinology \& Metabolism 100 2890-2898. (doi:10.1210/ JC.2015-2080)

Received in final form 29 April 2016

Accepted 12 May 2016 